\title{
Implementation of a New Method for an Improved Voltage Dips Evaluation by the Italian Power Quality Monitoring System in Presence of VT Saturation Effects
}

\author{
R. Chiumeo ${ }^{1}$, M . de Nigris ${ }^{1}$, L. Garbero ${ }^{1}$, C. Gandolfi ${ }^{1}, \underline{\text { L. Tenti }}^{1}$ and E. Carpaneto ${ }^{2}$ \\ ${ }^{1}$ ERSE - Enea Ricerche Sul Sistema Elettrico \\ Via Rubattino 54 - 20134 Milano (Italy) \\ Phone:+390239925126, Fax:+390239925557, e-mail: Riccardo.Chiumeo@erse-web.it, Michele.denigris@erse-web.it, \\ Luciano.Garbero@erse-web.it, Chiara.Gandolfi@erse-web.it, Liliana.Tenti@erse-web.it \\ ${ }^{2}$ Politecnico di Torino - Dipartimento di Energia Elettrica \\ C.so Duca degli Abruzzi 24 - 10129 Torino (Italy) \\ Phone:+390115647138, fax:+390115647199, e-mail: enrico.carpaneto@ polito.it
}

\begin{abstract}
The paper deals with a recent measurement improvement introduced into the Italian power quality monitoring system, concerning voltage dips detection and their proper evaluation in the presence of voltage transformer VT saturation phenomena. Saturation effects, essentially on network operated with isolated neutral, can give origin to "false" voltage dips, which can affect the on-line voltage monitoring during a few specific events (i.e. single line-to-ground faults). The "false dip" problem, on the other hand, can be considered negligible in compensated neutral networks.

A new criterion has been developed in cooperation with the Polytechnic of Turin, based on the detection of a second harmonic component in the measured voltages, which, once provided with the proper setting parameters, can succeed in identifying "false" dips. The criterion effectiveness has been evaluated on field. The major advantage of the filter implementation in the monitoring system is that, from now on, network operated with isolated neutral could be considered quite similar to those operated with neutral compensation from the point of view of voltage dip performance.
\end{abstract}

\section{Key words}

Power Quality, Monitoring System, Voltage Dips, Transformer Saturation, Distribution Networks

\section{Introduction}

The Italian power quality monitoring system QuEEN has been performing Power Quality (PQ) measurements in the MV distribution network since 2006 and on about 600 instruments distributed all over the Italian territory (400 units installed in HV/MV substations, 200 units installed along the MV lines ) [1].

The assessment of PQ performances across the country, during a long-term period campaign, could be very useful for regulation activity [2] provided that the system is periodically submitted to some indispensable technical updating from the point of view of both measurement techniques and data evaluation methods.

This paper deals with a recent measurement improvement introduced into the monitoring system, concerning voltage dips detection and their proper evaluation.

\section{Measuring chain features}

The QuEEN system makes use of existing voltage transformers (VT) in HV/MV substations and performs PQ measurements according to the international standard IEC EN 61000-4-30 [3]. Measurement Units (MUs) are connected to the LV side of voltage transformers, whose primary windings are connected between phase and ground. In this kind of system configuration a single phase-toground fault or line energization may cause the saturation of the voltage transformers giving place to the measure of "false" voltage dips which badly affect the dip-performance evaluation of the MV distribution network, especially in those networks operated with isolated neutral.

During faults in the MV network involving ground, a sinusoidal zero-sequence voltage arises. Its amplitude depends on fault type, fault location and fault resistance. Also a three-phase fault can cause zero-sequence voltage: after the arc extinction in the first phase, the fault involves two phases until the following extinctions. After the fault extinction, the zero-sequence voltage decays to zero with a behaviour depending on the neutral connection:

$\checkmark$ with compensated neutral (inductance tuned with the zero-sequence capacitance), the decay is a damped sinusoid at the resonance frequency, close to the fundamental frequency; the VT works properly; 
with isolated neutral, the decay is exponential and the VT can saturate; the saturation reduces the secondary voltages and a "false" voltage dip is observed.

Fig. 1 and Fig. 2 report two typical voltage dips monitored on field due to voltage transformer saturation whose main features are:

$\checkmark$ an asymmetrical waveform characterized by a two "camel humps" structure in the profile (Fig. 1); in the case of a "deep saturation" of the transformer (Fig. 2), a first symmetrical part, which is typical of a real 3 phase dip, followed by an asymmetrical one with the typical profile shown in the previous figure.

In these cases a specific software countermeasure is needed. The "false dip" problem can be considered negligible only in compensated neutral networks and do not concern MU placed along the MV lines because of the different voltage transformers network connection (primary winding line-toline connected).

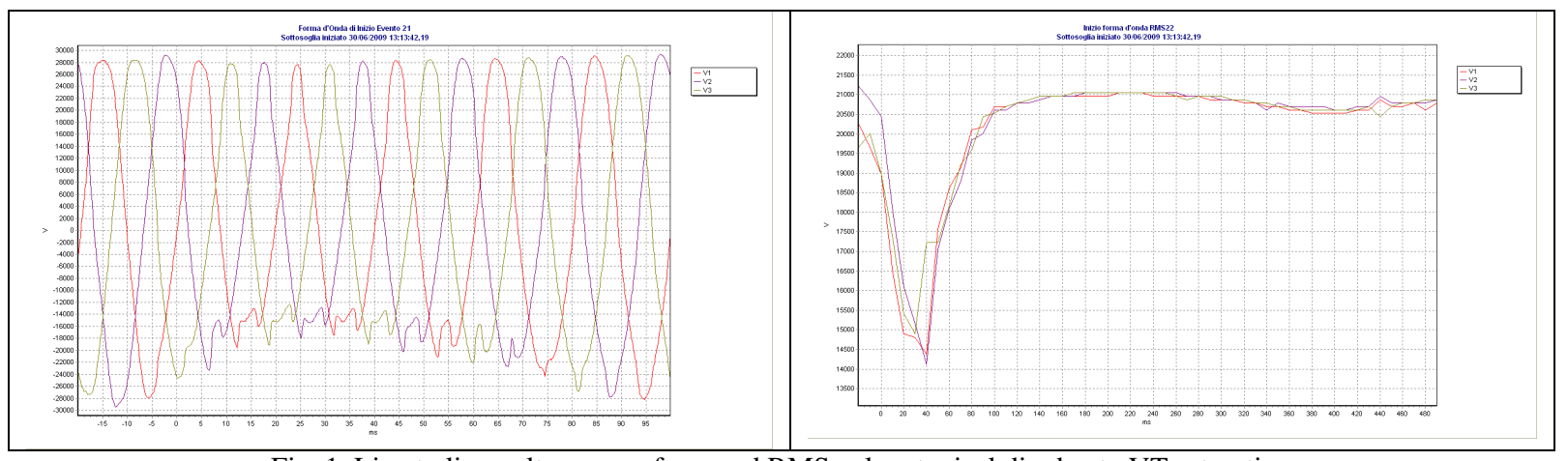

Fig. 1. Line to line voltage waveform and RMS value: typical dip due to VT saturation

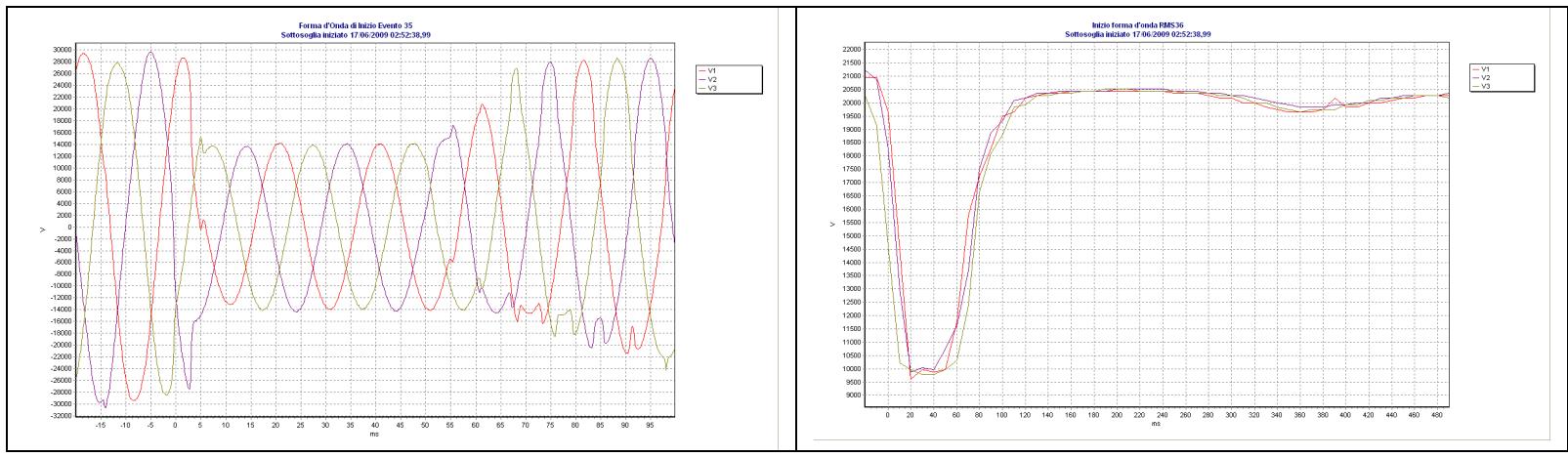

Fig. 2. Line to line voltage waveform and RMS value: typical dip due to a deep VT saturation

\section{A new criterion to overcome saturation effects}

In order to cope with this potential drawback, an advanced criterion, to distinguish "false" dips from the "real" ones, has been developed in cooperation with the Polytechnic of Turin. The criterion, based on the detection of a second harmonic component in the measured voltages, has been implemented in the QuEEN system on May 2009 after a simulation activity carried out by the Polytechnic in 2008 and a testing campaign performed at ERSE labs in Piacenza. The method replaces a former digital filter active in the system, based on the asymmetry of the measured voltage waveform.

Both partial VT saturations and exits from complete VT saturation can indeed give origin to a distorted voltage waveform characterized by a high $2^{\text {nd }}$ harmonic level.
This happens unfortunately also with real voltage dips just at their starting and ending points. An "effective" criterion has to distinguish between the two situations and give a specific evaluation for each event monitored.

In this frame the criterion developed classifies voltage dips into three types:

$\checkmark$ real ones

$\checkmark$ false ones

$\checkmark$ undefined; this last case corresponds to a not univocal evaluation of the event.

The $2^{\text {nd }}$ harmonic measurement is performed over $20 \mathrm{~ms}$ (to be refreshed every $10 \mathrm{~ms}$ ), as it happens with rms voltage measurements in general, in accordance with the standard 61000-4-30. The criterion relys on 2 parameters (a threshold for the $2^{\text {nd }}$ harmonic level and the minimum number of consecutive exceeding of the $2^{\text {nd }}$ harmonic threshold) and has to be applied independently to all the 
three phases. The lab campaign object has been to establish the best set of parameters for the final implementation of the criterion in the system, in order to recognize the greatest amount of false dips together with the fewest amounts of undefined situations and the minimum number of wrong classification for real dips. The choice of a particular set of parameters (a $2^{\text {nd }}$ harmonic threshold of $10 \%$ and three consecutive threshold exceeding, equivalent to $30 \mathrm{~ms}$ ) corresponds to the best balance of the above mentioned constraints for a correct false dips detection.

Both the calculation of the second harmonic level referred to a complete period and the use of a fix number of consecutive threshold exceeding, help to distinguish a false dip from a real one due to a fault, as at the beginning and at the end of the real dip a high $2^{\text {nd }}$ harmonic level is observed.

\section{Results}

Referring to about 7 weeks monitoring campaign and to the total number of dips monitored in this period, the $60 \%$ of the dips are classified as "real" events and the $28 \%$ as "false" ones by the criterion (Table 1). Subsequent analyses, spread over the year, have basically confirmed these first results.

Table I - New criterion Voltage dips evaluation

\begin{tabular}{|c|c|c|}
\hline $\begin{array}{c}\text { Voltage dips evaluation on the base of the new criterion } \\
\text { (7 weeks monitoring campaign) }\end{array}$ \\
\hline Type of dip & Number & \% \\
\hline Real dips & 6433 & 60 \\
\hline False dips & 3036 & 28 \\
\hline Undefined events & 1306 & 12 \\
\hline Total number & 10775 & 100 \\
\hline
\end{tabular}

The criterion effectiveness for the chosen set of parameters has been then established by the visual evaluation of the waveforms related to a certain amount of voltage dip events monitored during the defined period.

Being voltage dips characterized mainly by their duration $\mathrm{D}(\mathrm{ms})$ and residual voltage $\mathrm{U}_{\text {res }}$ (\% of rated voltage), contour maps of voltage dips number monitored have been drawn in the duration/residual voltage plane, just before and after the "filter" implementation.

Contour maps associated to "false" (Fig. 3) and "real" (Fig. 4) dips on the base of the new criterion confirm the assumption made in the past (before the new filter implementation) about the characterization of the two kinds of events from the point of view of duration and $\mathrm{U}_{\text {res. }}$.

The comparison of the contour maps obtained in the past [4] for compensated neutral networks with those drawn for the "real" dips confirms that the "false" dips problem can be considered negligible in such networks.

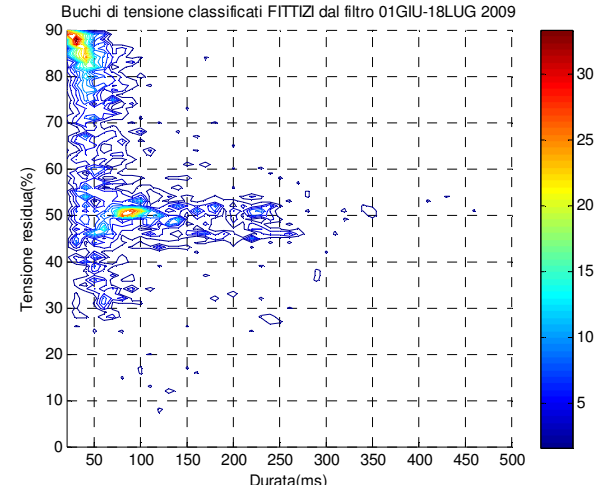

Fig. 3. Contours map for "false" dip number in the $D \backslash U_{\text {res }}$ plane

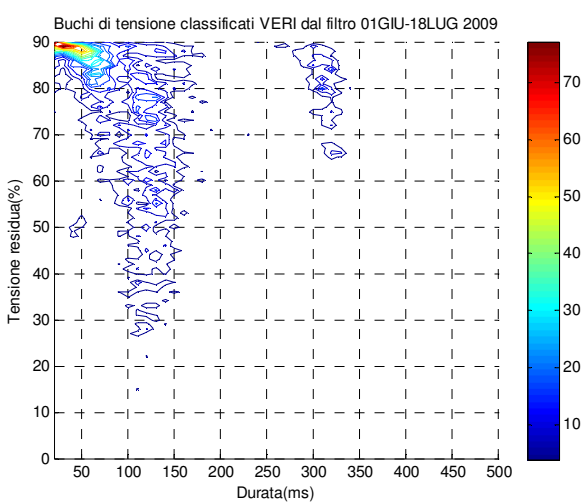

Fig. 4. Contours map for "real" dip number in the D $\backslash U_{\text {res }}$ plane

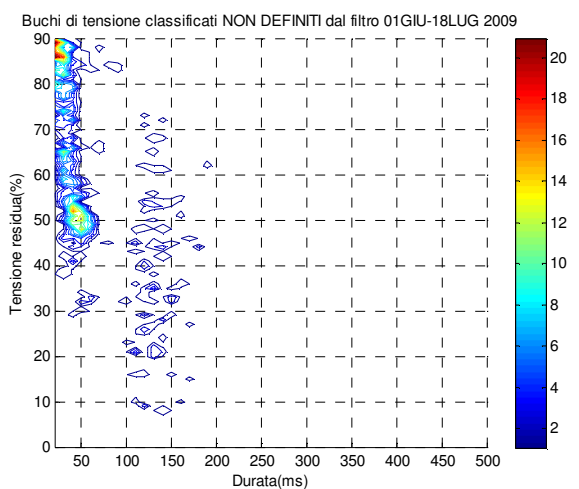

Fig. 5. Contours map for "undefined" event number in the $\mathrm{D} \backslash \mathrm{U}_{\text {res }}$ plane

As to undefined events (Fig. 5) they can be considered definitely "false dips" by visual inspection for duration classes less than $100 \mathrm{~ms}$. Others interesting results coming from waveform visual inspection of events characterized by a duration greater than $100 \mathrm{~ms}$, are:

$\checkmark$ the presence of a certain amount of "false" dips;

$\checkmark$ the presence of so called "multistage" events, which usually include both real and false events, due to polyphase grounded faults. Some simulations have been 
performed with ATP (Alternative Transient Program) to better investigate the phenomena (Fig. 7, Fig. 8).

At presents it seems difficult to think to a further filter improvement to be able to resolve these undefined multistage situations.

The criterion effectiveness in identifying properly voltage dips is influenced by the VT saturation conditions which can have effects both on voltage dip profile and duration.

For istance referring to the two false voltage dips shown in Fig 1 and Fig. 2 monitored on field, they have been classified in a different way by the criterion:

$\checkmark$ the former, correctly, as a false voltage dip;

$\checkmark$ the latter, being characterized by a complete VT saturation and, as a consequence, by a first part of the signal which appears perfectly sinusoidal, as a real dip.

Other cases which are very difficult to identify are:

$\checkmark \quad$ those characterized by a voltage dip duration which is not in compliance with the number of subsequent 2 nd harmonic threshold exceeding, as is the case with short duration voltage dips;

$\checkmark$ those due to grounded polyphase faults extinction, which usually appear as a sequence of real + false dip (Fig.6).

In the latter case the "false queue" appears at the fault extinction as it has been confirmed by some ATP simulations. In particular Fig. 7. Two phase grounded fault extinction: line to line simulated voltage at MV bus bar and the associated real voltage dip and Fig. 8 are relevant to a two phase grounded fault extinction $(180 \mathrm{~ms})$ :

$\checkmark$ Fig. 7 shows the line to line voltage waveform at MV bus bar and the "real dip" affecting the network;

$\checkmark$ Fig. 8 shows the same voltages measured at the VT secondary windings, highlighting the sequence real dip followed by the "false queue" due to saturation effect. In the figure the magenta curve refers to the dip identification signal. The presence of a voltage recovery before the two "camel humps" structure in Fig. 8 is not a false dip feature (Fig. 2).

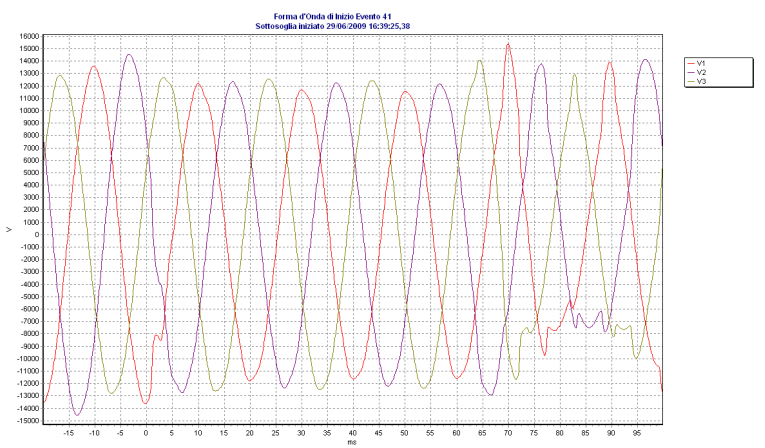

Fig. 6. On field measured line to line voltage waveform: real + false sequence

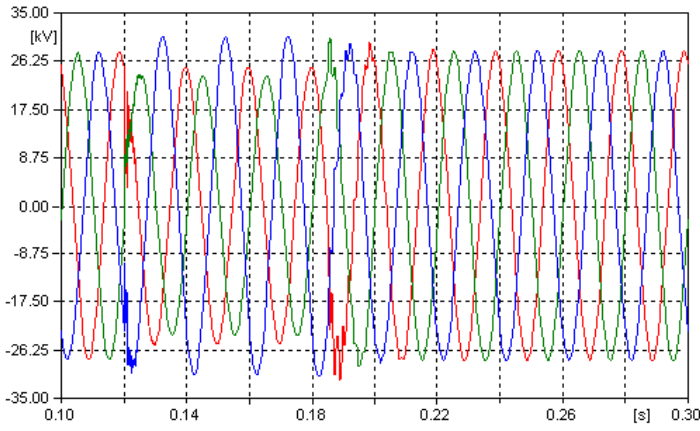

Fig. 7. Two phase grounded fault extinction: line to line simulated voltage at MV bus bar and the associated real voltage dip affecting the network

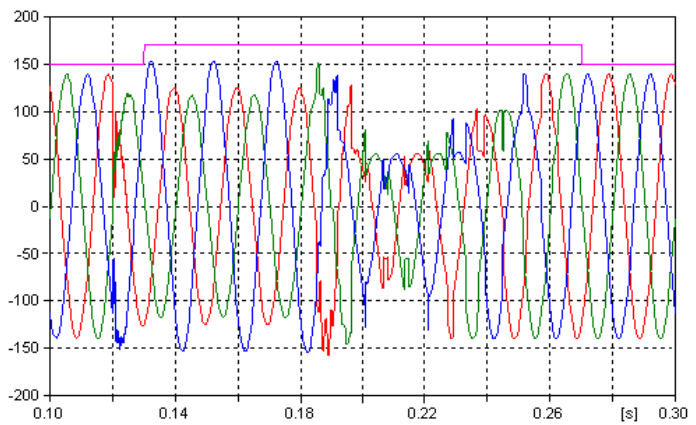

Fig. 8. Two phase grounded fault extinction $(180 \mathrm{~ms})$ : line to line voltage at the VT secondary side, dip identification signal (magenta curve). Real + "false queue"

\section{Conclusion}

The first advantage of the measurements improvement recently introduced in the Italian power quality monitoring system consists in having now a sound criterion which shows a better effectiveness in both false dip detection and their removal from voltage dip statistics, in comparison with the old filter still active before May 2009.

Another very important issue is that from now on neutral isolated networks can be considered similar to the compensated ones from the point of view of voltage dip performance. This is true for each duration and residual voltage classes which are no more interested by remarkable "false" dips pollution.

\section{Acknowledgement}

This work has been financed by the Research Fund for the Italian Electrical System under the Contract Agreement between CESI RICERCA and the Ministry of Economic Development-General Directorate for Energy and Mining Resources stipulated on June 21, 2007 in compliance with the Decree n.73 of June 18, 2007. 


\section{References}

[1]. F. Villa, A. Porrino, R. Chiumeo, S. Malgarotti, "The power quality monitoring of the MV network promoted by the Italian regulator", CIRED Paper 0042 (Wien 2007).

[2]. R. Chiumeo, A. Porrino, L. Garbero, L. Tenti , M. de Nigris, "The Italian Power Quality Monitoring System Of The MV Network: Results Of The Measurements Of Voltage Dips After 3 Years Campaign”, CIRED Paper 0737 (Prague 2008).

[3]. IEC 61000-4-30 - Electromagnetic Compatibility (EMC) Part 4-30: Testing and Measurement Techniques Power Quality Measurement Methods.

[4]. R. Chiumeo, A. Porrino, L. Garbero, L. Tenti, "Sistema di monitoraggio della qualità della tensione: evoluzione del sistema a supporto dell'attività regolatoria e delle proposte di revisione della normativa tecnica europea", Report CESI RICERCA 08005783 (Milano Febbraio 2009, http://erse-web.it) (Italian language). 